\title{
Design of Digital Campus Network Portal Platform Based on Portal
}

\author{
XIANG Haiyun ${ }^{1}$, FU Xiao ${ }^{2}$ \\ 1. Modern Educational Technology Center, Southwest Petroleum University, Si Chuan, ChengDu, \\ China, 610500 \\ 2. College of Computer Science, Southwest Petroleum University, Si Chuan, ChengDu, China, \\ 610500
}

Key words: portal system; digital campus; Portal

\begin{abstract}
The campus information portal based on Liferay is the entry point of the entire digital campus. It integrates the functions of the identity authentication and the user interface. The system can be integrated in the campus, so that users can easily publish and share information. Provides the service with other business flow management system, including the dynamic role binding, the role and the user group's authorization, the work task forwarding and so on functions, to a large extent increases the process flexibility, can be more appropriate to the actual, satisfies the user's need.
\end{abstract}

\section{Introduction}

Under the new form, the domestic colleges and universities in order to meet the needs of the times, have actively build and gradually build their own "Digital Campus" program. The digital campus is based on the network, the use of advanced information technology and tools, to achieve from the environment, resources, to the activities of all digital, on the basis of the traditional campus to build a digital space, to expand the time and space dimensions of the reality of the campus. There are many problems in the digital campus, mainly in several aspects, one is the lack of cooperation of each application system; the two is the lack of unified planning, information canot be effectively shared; the three is not to achieve data access between each other, the formation of information island; four is the user login system must be separated, no unified interface. Five is not a unified and effective planning, the entire digital campus is not easy to expand. Based on the above four points, the digital campus system users urgently need to build a unified portal, which can provide a unified service system, and can integrate the existing digital resources, provide personalized information services, to solve the problem of timely transmission, information sharing, collaborative processing business process.

\section{Principles of Portal}

\section{achieve the objectives and architecture}

The portal (Portal) platform is made of a series of technologies, Portal is a Web based application, which provides personalized, single sign on and different resource content aggregation, responsible for the performance of the entire information system. Aggregation is the behavior of different resources in a web page. A portal can have a lot of complex personality characteristics, to provide users with customized content. Portal pages have different portal components (Portlet), and have different contents for different users. Portal component (Portlet) is the most important concept in.Portlet, which is a Web based Portlet component, which is managed by Portlet, and the Portal container handles user requests and generates dynamic content. Portal takes Portlet as an available user interface component to provide a presentation layer for the information system.

Portal system seems to be exciting, in fact, there are a lot of problems to be faced, yet to be resolved. As the portal system is not to put some of the existing structured and unstructured information, documents listed in the portal system, but to integrate the portal system to achieve business and information automation. The concept and function that really want to take advantage 
of the complete portal system, I am afraid that all the network application system in the Department to rewrite the Java, and then one by one into the portal system.

Campus Portal CIP (Information) is a unified, open, open, information dissemination and sharing of information, and provide a wide range of applications, with high reliability, high availability, high performance, high security and high availability of the portal, and to provide services in the portal. Therefore, the portal must provide users with a unified information portal, and to provide users with safe and quick access to the required resources in any place. As a gateway to information access and management, the campus information portal is the best place to integrate campus applications and to provide users with a high efficiency and perfect environment. By integrating the campus application program into the portal, all users can be unified and continuous access to information. Users do not need to understand and do not need to care about the source of information, information is through the browser to the user.

The school currently through Portal, the existing application systems are integrated into the Portal. All of the new application system follows the JSR-168 standard, and can be integrated with the whole Portal very easily, and through WSRP to carry on the information exchange with the remote Portlet. Figure 1 shows the architecture of the Portal.

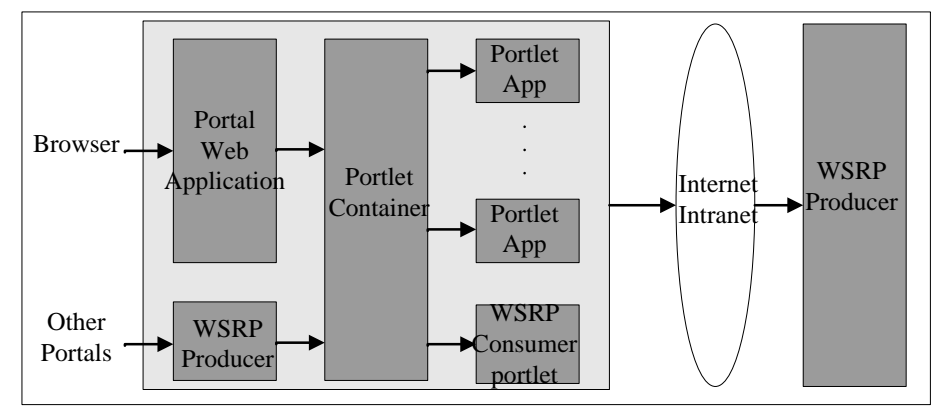

\section{specification and process}

Figure 1 Portal architecture diagram

Java Standardization Request168 (JSR 168) specification and WSRP standards (Service for Remote Portlets Web), these two standards include a very good programming specification, and released the open source code, the source code to achieve the appropriate definition of all the necessary functions, can be a good collaboration as the pursuit of the goal.

Jsr168 standard (Java specification request) is released by the JCP (Java community process) organization to provide interoperability between different portal and portlet. Using JSR168PortletAPI development of a portal, you can run in all support for this standard portal server, so that portal applications can use different vendor portal server.

To solve the problem of Portal OASIS Remote Portlet1.0 standard, [8] organization has the Web Services for WSRP: standard, which can be used to make use of the product characteristics of different portal server, and WSRP can call the remote Portlet service, which makes the communication between enterprise and partner more convenient and fast.

\section{Portal Framework Design}

Unified output mode, the information of the sub level, sub authority, personalized output. Extranet portal resources are divided into structured data, unstructured data, application system by the corresponding access gateway and an integrated package technology to access information resources for centralized registration issued, all kinds of application system integration, the campus users the freedom to customize personalized information content. A unified directory management for all kinds of resources, the classification of all users, group management. Based on the unified directory management system, the authentication and authorization can be realized between the user and the program. Unified portal platform in the entire digital campus to provide users with a variety of campus information single access point of service, namely unified identity authentication and single point landing, through the campus unified information portal provides comprehensive information and services, users can use within a specific authority within the application subsystem 
or access to the corresponding data information. Through the powerful personalized portal, the school teachers and students can quickly and easily find and access to a variety of resources to meet the needs of the completion of the course or project.

In the face of the existing scattered application system, we take the method is to build a unified information portal for information and resources integration, in Portal, Portlet is not just as a small window, but also a kind of application, is a tool for business integration, so we have a good information integration and application integration platform. For example, a NwesPortlet can complete the collection of information, list the most important news, information retrieval and other functions: so, with the Portlet for different business applications, users can customize their own portal according to their own authority, from a unified interface to access information, online interaction, with other people to work together to complete the entire application system access.

Portlet development has a variety of forms, can be JSP, Struts and custom framework class. For simple portlet, the general use of JSP form. This system uses Struts as the main way to realize the portlet. In order to be easy to develop and expand, most of the portlet in this system is implemented by using a custom framework. The custom frame is the advanced form of JSP. You need to customize a framework class, each of the links to the portlet framework class is responsible for the shift. Key code of the framework class is as follows:

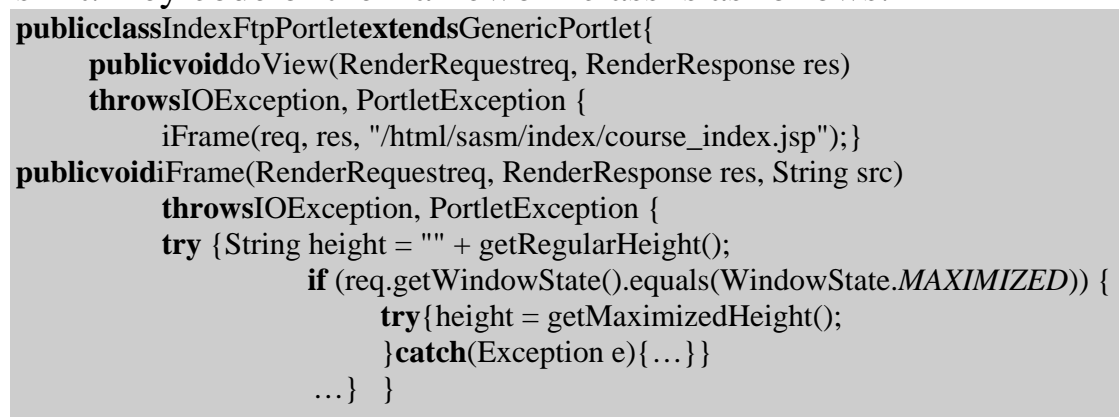

In order to develop a convenient, improve the degree of reuse of the program, in the definition of a major type of framework, the other portlet class are inherited from the parent frame class, only to change the portlet of the sub class.

\section{Summary}

Digital campus information portal service provides the service of the traditional campus network. Construction of the digital campus electronic school affairs foundation platform through the portlet technology unified portal platform, integration of existing information systems and web applications. The design supports the integration of data, content and information, and through the use of the available channels to deliver the inheritance of content, applications and services, reduce the operating costs of the campus information portal, improve the efficiency of the user's work, in the construction of the digital campus has an important role. The design of the portal system has been applied to the digital campus system, and it has some feasibility and practicality.

\section{Reference}

[1] Mingda Zhou, GuanqunBao, YishuangGeng, Bader Alkandari, Xiaoxi Li, Polyp detection and radius measurement in small intestine using video capsule endoscopy, 2014 7th International Conference on Biomedical Engineering and Informatics (BMEI), Oct. 2014

[2] Gan Yan, YuxiangLv, Qiyin Wang, YishuangGeng, Routing algorithm based on delay rate in wireless cognitive radio network, Journal of Networks, 9(4), 948-955, Jan. 2014

[3] GuanqunBao, Liang Mi, YishuangGeng, KavehPahlavan, A computer vision based speed estimation technique for localizing the wireless capsule endoscope inside small intestine, 36th Annual International Conference of the IEEE Engineering in Medicine and Biology Society (EMBC), Aug. 2014 
[4] Xinchao Song, YishuangGeng, Distributed community detection optimization algorithm for complex networks, Journal of Networks, 9(10), 2758-2765, Jan. 2014 